\title{
Organização didático-metodológica das aulas de Anatomia e Fisiologia Humana: comportamento e percepção dos estudantes
}

\section{Didactic-methodological organization of Human Anatomy and Physiology classes: students' behavior and perception}

\author{
Aline Marian Callegaro ${ }^{1}$ \\ Karla Marques da Rocha ${ }^{2}$
}

\begin{abstract}
RESUMO
O presente artigo apresenta os resultados de uma pesquisa quantitativa, do tipo pesquisa-ação, cujo objetivo foi analisar o comportamento e a percepção dos estudantes sobre a organização didático-metodológica, através dos momentos didático-problematizadores, implementada em três aulas da Disciplina de Anatomia e Fisiologia Humana de um Curso Técnico em Enfermagem da cidade de Santa Maria, no estado do Rio Grande do Sul, Brasil. Foram planejadas pelas pesquisadoras três aulas sobre os temas: sistema endócrino, tegumentar e nervoso, tendo por base a organização didático-metodológica através dos momentos didático-problematizadores. As aulas foram implementadas numa turma do Módulo I, do Curso Técnico em Enfermagem, constituída por oito estudantes. Para a coleta dos dados foram utilizados diário de bordo para registro dos comportamentos dos estudantes observados em cada aula e questionário com perguntas fechadas e abertas para identificar a percepção e sugestões dos estudantes acerca da organização didático-metodológica através dos momentos didático-problematizadores. Dentre os principais resultados, destaca-se a percepção da maioria dos
\end{abstract}

DOI: $10.1590 / 0104-4060.42422$

1 Universidade Federal do Rio Grande do Sul. Porto Alegre, Rio Grande do Sul, Brasil. Rua Sarmento Leite, n 521. CEP: 90050-170.E-mail: nimacall@gmail.com

2 Universidade Federal de Santa Maria. Santa Maria, Rio Grande do Sul, Brasil. Av. Roraima, nº 1.000. Camobi. CEP: 97105-900.E-mail:karlamarquesdarocha@gmail.com 
estudantes que considerou boa e/ou muito boa a organização didático-metodológica utilizada, assim como os seus momentos constituintes (desafio inicial, melhor solução escolar no momento, desafio mais amplo e tarefa extraclasse). Sendo que alguns estudantes ainda apresentam comportamento passivo diante do processo de ensino-aprendizagem, concluindo-se que ainda existe uma necessidade de mudanças culturais neste contexto pesquisado, partindo especialmente das ações docentes.

Palavras-chave: organização didático-metodológica; momentos didático-problematizadores; Disciplina de Anatomia e Fisiologia Humana; Curso Técnico em Enfermagem.

\begin{abstract}
This paper presents the results of a quantitative research, a kind of action research which analyzed the students' behavior and perceptions about the didactic-methodological organization, through problem-solving didactic moments implemented in three classes of the Human Anatomy and Physiology Discipline of a Technical Course in Nursing in Santa Maria, Rio Grande do Sul, Brazil. The researchers planned three classes with the following topics: endocrine, cutaneous and nervous systems, based on the didactic-methodological organization, through problem-solving didactic moments. The lessons were implemented in a class of Module I of the Technical Course in Nursing that consists of eight students. The data collecting was performed through a logbook to record the observed students' behavior in each class and a questionnaire with closed and open questions to identify the students' perceptions and their suggestions about the didactic-methodological organization through the problem-solving moments. Among the main results, there is the majority of students' perception that considered the didactic-methodological organization good and/or very good as well as its constituent moments (initial challenge, the best school solution at the time, broader challenges and extra classroom tasks). Some students still have passive behavior on the teaching-learning process. Thus, there is still a need for cultural changes studied in this context, especially starting by teachers' actions.
\end{abstract}

Keywords: didactic-methodological organization; problem-solving didactic moments; Human Anatomy and Physiology Discipline; Technical Course in Nursing. 


\section{Introdução}

$\mathrm{Na}$ época da influência comportamentalista, o ensino e a aprendizagem possuíam enfoques em estímulos, respostas e reforços, não em significados do contexto educativo. Hoje, o discurso está embasado na aprendizagem significativa, mudança conceitual e construtivismo. Para Freire (1999), a posição normal do homem não é apenas estar no mundo, mas estar com ele, desenvolver relações com a realidade que resultam no conhecimento.

A pedagogia da problematização ou educação para o pensar é fundamentada na pedagogia da autonomia. Ela propõe a construção do conhecimento pelo movimento de agir sobre a realidade. Nesta perspectiva, a realidade é refeita pela reflexão que gera conscientização e orienta o sujeito para a transformação por meio da práxis. (FREIRE, 2006).

Possolli (2009) afirma que a construção de políticas e projetos da Educação Profissional deve ser norteada pela educação para o pensar, currículo por competência e pedagogia de projetos, interdisciplinaridade e transversalidade, vinculação com as mudanças no mundo do trabalho e novas tecnologias aplicadas à Educação Profissional. Para atender às necessidades da Educação Profissional é preciso conhecer algumas teorias educacionais para embasar a ação docente e, especialmente, a organização didático-metodológica das aulas.

A partir dessas colocações, surge uma possível questão de pesquisa: qual é o comportamento e a percepção dos estudantes acerca de uma organização didático-metodológica via os momentos didático-problematizadores utilizada em aulas da Disciplina de Anatomia e Fisiologia Humana de um Curso Técnico em Enfermagem, da cidade de Santa Maria, Rio Grande do Sul (RS)? A resolução deste problema de pesquisa permitirá uma melhor compreensão da realidade dos estudantes e, mais especificamente, de seus comportamentos e percepções frente à abordagem didático-metodológica e do processo de ensino-aprendizagem estabelecido na Disciplina em estudo.

Assim, o presente artigo tem como objetivo analisar o comportamento e a percepção dos estudantes sobre a organização didático-metodológica através dos momentos didático-problematizadores das aulas da Disciplina de Anatomia e Fisiologia Humana, de um Curso Técnico em Enfermagem. Para atingir o objetivo geral foram propostos os seguintes objetivos específicos: $(i)$ conhecer e analisar o comportamento dos estudantes sobre as aulas em que se utilizou a organização didático-metodológica via os momentos problematizadores; (ii) analisar a percepção dos estudantes sobre esta organização e o processo de 
ensino-aprendizagem estabelecido; assim como (iii) as sugestões dos estudantes acerca desta organização didático-metodológica.

A estrutura do artigo compreende: a seção 2, onde se apresenta o referencial teórico acerca do problema de pesquisa; a seção 3, onde constam os procedimentos metodológicos; a seção 4 , com os resultados e discussões; e a seção 5, que apresenta as considerações finais do estudo.

\section{Referencial teórico}

Um dos objetivos mais amplos da educação é oportunizar o exercício pleno da cidadania. Para isso um mínimo de formação básica em Ciência é fundamental para a formação cultural de qualquer cidadão. $\mathrm{O}$ conhecimento científico não pode ser administrado numa perspectiva de simples transmissão, mas numa abordagem crítica, que caracteriza o empreendimento científico como uma atividade humana. (DELIZOICOV; ANGOTTI, 1994).

A concepção educacional Freireana, mais especificamente a pedagogia da problematização, propõe a construção do conhecimento pelo movimento de agir sobre a realidade. (FREIRE, 2006). Dentro desta perspectiva, pode-se considerar a aula como um microcosmo dinâmico, conectado ao currículo de formação. A ação de ensino da qual resulta a aprendizagem deve superar a exposição tradicional de conteúdos e o simples dizer do conteúdo por parte do professor (ANASTASIOU, 2003); conhecer os estudantes, seus comportamentos, percepções, e diferentes abordagens didático-metodológicas que possibilitam o melhor processo de ensino-aprendizagem. (MELLO, 2007).

Berbel (1998) complementa que metodologias de ensino baseadas na problematização podem ser utilizadas sempre que oportuno, quando os temas são relacionados à vida em sociedade. Pois elas requerem basicamente alterações na postura do professor para a organização da disciplina, desenvolvimento interativo, reflexivo e crítico dos temas com os estudantes. Da mesma forma, podem ser utilizadas abordagens didático-metodológicas que, segundo Masetto (2003/2004), estimulem o aluno a apreender participando do processo de ensino-aprendizagem.

Uma possível metodologia baseada na problematização foi proposta por Delizoicov e Angotti (1994) para o ensino de Ciências envolvendo três momentos pedagógicos: problematização inicial, organização do conhecimento científico e aplicação do conhecimento. Mallmann, Bastos e Müller (2004) propõem uma organização didático-metodológica, pensada como guia para a operacionaliza- 
ção da ação escolar via momentos didáticos-problematizadores, que são três: desafio inicial (DI), melhor solução escolar no momento (MSEM) e desafio mais amplo (DA). Os quais, no pensar de Abegg e Bastos (2005), possuem as seguintes características:

a) DI - os estudantes são envolvidos ativamente na resolução de um desafio concreto ou problema, proposto pelo professor e referente ao recorte temático da aula. Não são fornecidas respostas às dúvidas dos estudantes neste primeiro momento, mas o professor explicita o significado que tem para a aula o desafio proposto, através da problematização dos entornos da situação desafiadora;

b) MSEM - problematizam-se as visões de mundo iniciais dos estudantes frente ao conhecimento científico. Neste momento a problematização realizada pelo professor é compreendida a partir dos conhecimentos científicos e tecnológicos disponíveis e necessários para a resolução do desafio, organizados em torno de uma rede conceitual elaborada, relacionada ao desafio inicial. São também trabalhadas as contradições explicitadas pelas visões de mundo dos estudantes com os conhecimentos científicos no intuito de superar as "situações-limite" identificadas no primeiro momento;

c) DA - outro desafio é proposto, que se relaciona ao inicial de alguma maneira, mas, neste momento, com o objetivo dos estudantes operacionalizarem os principais conceitos abordados. É quando se pode avaliar processualmente a aprendizagem dos conhecimentos científicos e tecnológicos abordados na aula, pois a resolução da situação-problema proposta requer a operacionalização e avaliação dos conhecimentos escolares problematizados na aula. Desta forma, têm-se elementos de avaliação do aprendizado do estudante, assim como, do processo de desenvolvimento da aula.

Os autores anteriores complementam os momentos com a possibilidade de realização da tarefa extraclasse (TE), em que o professor pode propor uma tarefa para casa ao final das aulas, visando à retomada pelo estudante dos conceitos abordados no encontro presencial.

\section{Procedimentos metodológicos}

Esta pesquisa é qualiquantitativa, do tipo pesquisa-ação. Um tipo de pesquisa social, concebida e realizada em associação com uma ação do pesquisador, que possui uma metodologia pedagogicamente estruturada. Os pesquisadores e os participantes representativos da situação ou problema estão envolvidos de modo cooperativo ou participativo. (FRANCO, 2005). 
O cenário do estudo compreende uma turma constituída por oito estudantes matriculados na Disciplina de Anatomia e Fisiologia Humana do Módulo I, de um Curso Técnico em Enfermagem, de uma Instituição privada da cidade de Santa Maria, no estado do Rio Grande do Sul, Brasil. Nesta turma uma das pesquisadoras realizou o Estágio Supervisionado III do Programa Especial de Graduação de Formação de Professores para a Educação Profissional da Universidade Federal de Santa Maria. O estudo foi realizado no período de dois meses - outubro e novembro de 2011 - e os oito estudantes, com idades entre 26 a 50 anos, participaram da pesquisa e oficializaram seu aceite através da assinatura do Termo de Consentimento Livre Esclarecido (TCLE).

Inicialmente foram planejadas pelas pesquisadoras três aulas sobre os temas: sistema endócrino, tegumentar e nervoso, tendo por base a organização didático-metodológica através dos momentos didático-problematizadores. (ABEGG; BASTOS, 2005). As aulas planejadas foram implementadas em três dias diferentes.

A coleta dos dados foi realizada em diário de bordo e questionário. Sendo que, no final de cada aula, foram realizadas considerações e análise das implementações no diário de bordo, enfatizando-se, especialmente, as observações sobre o comportamento dos estudantes. Para suportar a posterior análise foram realizados inclusive registros sistemáticos do(a): (i) visão geral da aula; (ii) descrição detalhada de acontecimentos significativos; (iii) tipo de comportamento dos estudantes; (iv) frases textuais do professor ou dos estudantes; (v) dúvidas, contradições, reflexões que surgiram durante ou após o desenvolvimento das atividades. Cabe destacar que o diário de bordo propicia o desenvolvimento dos níveis descritivos, analítico-explicativos, valorativos e reflexivos do processo de investigação. (PORLÁN; MARTIN, 1997).

No final das três aulas foi aplicado um questionário aos seis estudantes presentes para identificar suas percepções acerca da organização didático-metodológica das aulas implementadas. O questionário constou de nove perguntas abertas e fechadas:

a) seis questões de múltipla escolha com escala ordinal em que os estudantes avaliaram a afirmativa de muito ruim a muito boa, sendo que havia opções intermediárias: ruim, regular e bom. Havia também uma parte descritiva para sugestões. Estas questões visaram especificamente identificar a percepção dos estudantes acerca: (i) das aulas implementadas; (ii) da organização didático-metodológica via momentos didático-problematizadores; (iii) DIs; (iv) MSEMs; (v) DAs; e (vi) TEs;

b) uma questão binária (opções de sim ou não) também com parte descritiva para sugestões para identificar se o estudante acredita que a organização 
didático-metodológica via os momentos didático-problematizadores facilitou a compreensão e aprendizado dos conteúdos;

c) duas questões descritivas para: (i) investigar se outras diferentes organizações didático-metodológicas poderiam facilitar o processo de ensino-aprendizagem do estudante; e (ii) identificar com qual organização o estudante aprende melhor.

O conteúdo do diário de bordo e a parte qualitativa dos questionários foram submetidos a uma análise de conteúdo. (BARDIN, 2011). Tanto a parte quantitativa quanto a qualitativa do questionário foram descritas e interpretadas. Os resultados são apresentados e discutidos na próxima seção.

\section{Resultados e discussões}

Nesta seção são apresentados os resultados, integrando-se a análise dos achados do diário de bordo às partes quantitativa e qualitativa do questionário. Os resultados são discutidos com outros estudos científicos relacionados ao tema.

A turma em que foi realizada a pesquisa era do Módulo I, do Curso Técnico em Enfermagem, constituída por oito estudantes matriculados na Disciplina de Anatomia e Fisiologia Humana: sete do sexo feminino e um do sexo masculino, com faixa etária entre 26 a 50 anos. Seis responderam ao questionário: cinco do sexo feminino e um do sexo masculino.

Inicialmente se buscou ter uma visão geral das aulas implementadas, para isso foram analisados o comportamento e as percepções gerais dos estudantes em relação às aulas. Segundo as observações do diário de bordo, a maioria dos estudantes foi a todas as aulas, realizaram as atividades propostas (com algumas reclamações discutidas na sequência) e dialogaram durante as implementações. Segundo as informações do questionário se identificou que $50 \%$ dos participantes consideraram as aulas muito boas; cerca de $33 \%$, boas; e aproximadamente $17 \%$, regulares. Nenhum estudante avaliou as aulas como ruins ou muito ruins, nem mesmo realizou sugestões. Quando se investigou a opinião geral dos estudantes sobre a organização didático-metodológica através dos momentos didático-problematizadores, identificou-se que aproximadamente $33 \%$ avaliaram como regular e cerca de $67 \%$ como muito boa. Um dos participantes, que considerou regular, sugeriu que as aulas fossem comentadas apenas no final, quando já tivesse conhecimento do conteúdo.

Esta sugestão preocupa e demonstra que nem todos os estudantes se propõem a assumir uma postura ativa no seu processo de ensino-aprendizagem, 
restringindo o diálogo, defendido por Freire (1999) como um recurso para inserir o homem na sociedade e adotado na organização didático-metodológica das aulas implementadas. Anastasiou (2003) afirma que uma parceria deliberada e consciente entre os sujeitos (professor e estudante) é necessária para efetivar a construção do conhecimento escolar. Moreira et al. (1997) ressaltam que os processos interativos em sala de aula motivam o desenvolvimento de inúmeras formas de "aprender a aprender", que contribuem para a construção do conhecimento. Auxiliando inclusive no desenvolvimento das habilidades de reflexão, interação e prática.

Quando analisados cada momento didático-problematizador da organização proposta, o comportamento e a percepção dos estudantes sobre o DI, DA e TE foi semelhante: cerca de $33 \%$ dos participantes consideraram bons e aproximadamente $67 \%$, muito bons. Percepções que surpreenderam, pois segundo dados do comportamento dos estudantes apontados no diário de bordo e, até mesmo pela própria análise geral das percepções sobre a organização didático-metodológica realizada anteriormente, alguns estudantes reclamaram especialmente para realizar o DI, pois era o primeiro momento da aula e preferiam adotar uma postura mais passiva. Quanto à opinião da maioria, aproximadamente $67 \%$, pode-se sugerir que suas percepções modificaram-se no decorrer do período de implementação das aulas, indicando que o diálogo adotado em aula, especialmente em relação à importância de participar ativamente no processo de ensino-aprendizagem, foi importante para os estudantes.

Logo, estes resultados enfatizam a importância da organização reflexiva das aulas (FREIRE, 1999) e se assemelham às considerações de Marin et al. (2010) de que as metodologias ativas de aprendizagem, ancoradas na criticidade, são entendidas como o modelo de formação profissional mais condizente com os princípios da atual política de saúde. O curso que utiliza metodologias ativas possibilita, além dos ganhos intelectuais, a articulação entre pensar, sentir e agir, envolvendo a integralidade, a articulação ensino-serviço e o enfrentamento das complexas e diversificadas situações do cotidiano dos serviços de saúde.

A análise do MSEM apresentou resultados diferenciados dos demais momentos didático-problematizadores. Aproximadamente 50\% avaliou o MSEM como muito bom, cerca de 33\% como bom e 17\% como regular. Quantitativamente igual a avaliação geral das aulas. Demonstrando que o momento de problematização dos conhecimentos científicos foi satisfatório para cerca de $83 \%$ dos participantes e não satisfatório para nenhum dos estudantes. O que se confirma com os dados do diário de bordo, no qual consta que boa parte dos estudantes apresentaram interesse pelo MSEM e interagiram durante este momento.

Além disso, cerca de $67 \%$ dos estudantes que reponderam ao questionário acreditam que a organização didático-metodológica, via os momentos didático- 
problematizadores, facilita a compreensão e o aprendizado dos conteúdos, em relação aos aproximados 33\% que discordaram. Dois dos indivíduos consideraram esta organização didático-metodológica utilizada como a melhor. Dentre as outras formas de organizar as aulas (diferentes desta utilizada), que poderiam facilitar o processo de ensino-aprendizagem citadas pelos estudantes, encontra-se exclusivamente o seguimento da apostila desenvolvida pelo professor da disciplina - opinião de quatro dos estudantes. Um deles complementou, sugerindo que não fossem misturados os conteúdos e, de acordo com outro participante, "quando se pega um conteúdo em andamento os alunos já estão acostumados com o outro professor e se deve seguir a forma de ensino dele", ou seja, com a organização que segue a leitura comentada da apostila.

Resultados semelhantes foram identificados quando se analisa a organização de aula em que cada estudante julga aprender melhor. Pois se destacam: leitura e explicação, acompanhamento da apostila - quatro estudantes a sugeriram, porém um deles ressaltou que as explicações do professor também são importantes.

Estes últimos resultados novamente são confirmados pelas observações do diário de bordo, especialmente do primeiro dia de implementação da aula, em que se percebeu que os estudantes estavam habituados com uma organização didático-metodológica que não incentivava a postura ativa, o diálogo, a participação e a criticidade. Constatações semelhantes e talvez até justificadas pelo estudo de Damasceno e Cória-Sabini (2003), que elucida que os estudantes das profissões biomédicas são normalmente encorajados a aprender por meio da memorização e fixação de conteúdos, sem o devido suporte teórico e questionamento crítico.

Estes mesmos autores enfatizam que os professores devem ir além da apresentação da disciplina como Ciência apoiada em método científico próprio e criar recursos didáticos para que os estudantes desenvolvam estudos independentes. Postura necessária para um profissional de uma área em que as inovações tecnológicas ocorrem num ritmo bastante acelerado.

Para auxiliar na modificação desta realidade, Montes e Souza (2010) afirmam que é importante a interação professor-aluno num processo contínuo de adaptação e de evolução para o aprendizado significativo. Sendo primordial auxiliar o aluno a organizar sua estrutura cognitiva para se tornar autor de seu próprio aprendizado. $\mathrm{O}$ que pode possibilitar a modificação desta realidade retratada pelo presente estudo. Pois, conforme Mello (2007), mesmo que cada professor possua sua própria metodologia, que não é única e acabada, ele deve encontrar a organização didático-metodológica apropriada para cada situação, oportunizando o diálogo em sala de aula. 


\section{Considerações finais}

Por meio de resultados do estudo, uma pesquisa qualiquantitativa, do tipo pesquisa-ação, cujas coletas dos dados foram por diário de bordo e questionário, respondeu-se a questão de pesquisa do estudo, ou seja: Qual seria o comportamento e a percepção dos estudantes acerca da organização didático-metodológica através dos momentos didático-problematizadores das aulas da Disciplina de Anatomia e Fisiologia Humana, do Módulo I, de um Curso Técnico em Enfermagem, da cidade de Santa Maria, RS? Pois foi realizada a análise do comportamento e percepção dos estudantes deste contexto proposto e, consequentemente, atingidos o objetivo geral e os objetivos específicos, pois se identificou que o comportamento e especialmente a percepção da maioria dos estudantes indicaram que a organização proposta e seus momentos foram bons e/ou muito bons. Sendo que alguns deles ainda preferem adotar postura passiva frente ao processo de ensino-aprendizagem.

Alguns estudantes apresentam comportamento passivo diante do processo de ensino-aprendizagem e hesitam em adotar uma postura ativa, reflexiva e crítica, que exceda a leitura comentada da apostila, organização didático-metodológica preferida pela maioria. Assim, cabe considerar que o professor, sujeito do seu próprio trabalho e autor de sua pedagogia, precisa refletir sobre seus objetivos e abordagens didático-metodológicas adotadas nas aulas. Buscando incentivar o diálogo, a reflexão e a criticidade dos estudantes para que eles sejam autores de seu próprio conhecimento e consigam resolver diferentes possíveis problemas da vida em sociedade.

Como possibilidade de pesquisas futuras, sugere-se o estudo das abordagens didático-metodológicas adotadas por docentes do ensino técnico, assim como o estudo de comportamento e percepção de outros estudantes sobre esta mesma organização investigada. Podendo-se analisar ainda a implementação de outras organizações didático-metodológicas, sob diversos aspectos e contextos.

\section{REFERÊNCIAS}

ABEGG, I.; BASTOS, F. P. Fundamentos para uma prática de ensino-investigativa em Ciências Naturais e suas tecnologias: exemplar de uma experiência em séries iniciais. Revista Electrónica de Enseñanza de las Ciencias, v. 4, n. 3, 2005. Disponível em: 
$<$ http://www.saum.uvigo.es/reec/volumenes/volumen4/art7_vol4_n3.pdf >.Acesso em: 13 maio 2012.

ANASTASIOU, L. G. C. Ensinar, aprender e processos de ensinagem. 2003. Disponível em: <www.fcf.usp.br/Ensino/Graduacao/Disciplinas/Exclusivo/Inserir/Anexos/LinkAnexos/CAPÍTULO\%201\%20LeaAnastasiou.pdf>. Acesso em: 23 jun. 2011.

BARDIN, L. Análise de conteúdo. São Paulo: Edições 70, 2011.

BERBEL, N. N. "Problematization" and Problem-Based Learning: different words or different ways? Interface - Comunicação, Saúde, Educação, Botucatu, v. 2, n. 2, p. 139-154, 1998.

DAMASCENO, S. A. N.; CÓRIA-SABINI, M. A. Ensinar e aprender: saberes e práticas de professores de Anatomia Humana. Revista de Psicopedagogia, São Paulo, v. 20, n. 63, p. 243-54, 2003.

DELIZOICOV, D.; ANGOTTI, J. A. Metodologia do ensino de Ciências. 2. ed. São Paulo: Cortez, 1994.

FRANCO, M. A. S. Pedagogia da Pesquisa-Ação. Educação e Pesquisa, São Paulo, v. 31, n. 3, p. 483-502, set./dez. 2005.

FREIRE, P. Educação como prática da liberdade. 23. ed. Rio de Janeiro: Paz e Terra, 1999.

FREIRE, P. Pedagogia da Autonomia: saberes necessários à prática educativa. 34. ed. Rio de Janeiro: Paz e Terra, 2006.

MALlmanN, E. M.; BASTOS, F. P.; MÜLLER, F. M. Problematização do conteúdo-metodologia como estratégia para fortalecer o diálogo-problematizador. 2004. Disponível em: <http://amem.ce.ufsm.br>. Acesso em: 04 dez. 2011.

MARIN, M. J. S. et al. Multiprofissional health-related graduate courses: results from experiences using active methodologies. Interface - Comunicação, Saúde, Educação, Botucatu, v. 14, n. 33, p. 331-344, abr./jun. 2010.

MASETTO, M. T. Inovação na educação superior. Interface - Comunicação, Saúde, Educação, Botucatu, v. 8, n. 14, p. 197-202, set. 2003/fev. 2004.

MELLO, E. A relação com o saber e a relação com o ensinar no estágio supervisionado em Biologia. Dissertação (Programa de Mestrado em Ensino de Ciências e Educação Matemática) - Universidade Estadual de Londrina, Londrina, 2007.

MONTES, M. A. A.; SOUZA, C. T. V. Estratégia de ensino-aprendizagem de anatomia humana para acadêmicos de medicina. Ciência e Cognição, Rio de Janeiro, v. 15, n. 3, p. 002-012, 2010.

MOREIRA, M. A. Teorias de Aprendizagem. São Paulo: Pedagógica e Universitária, 1999. 
MOREIRA, A. M. et. al. A prática de ensino e a produção de saberes na escola. Rio de Janeiro: DP\&A, 1997.

PORLÁN, R.; MARTIN, J. El diário del professor: un recurso para la investigación en el aula. Sevilla: Díada, 1997.

POSSOLLI, G. E. Políticas educacionais e seus agentes definidores: pressupostos para a definição de políticas para a educação profissional. Educação Profissional: Ciência e Tecnologia, Brasília, v. 3, n. 2, p. 237-247, jan./jun. 2009.

Texto recebido em 05 de agosto de 2015. Texto aprovado em 13 de dezembro de 2015. 